\title{
Thermodynamic Properties of Concentrated Solutions of Poly(hexyl isocyanate), a Liquid Crystalline Polymer
}

\author{
Takashi Itou, Takahiro SATo, ${ }^{\dagger}$ Akio Teramoto, \\ and Shaul M. AHARONI* \\ Department of Macromolecular Science, Osaka University, \\ Toyonaka, Osaka 560, Japan \\ * Allied-Signal Corporation, Engineered Materials Sector Laboratories, \\ New Jersey 07960, U.S.A.
}

(Received June 7, 1988)

\begin{abstract}
Osmotic pressure and sedimentation equilibrium measurements were made on isotropic solutions of poly(hexyl isocyanate), a stiff-chain polymer forming a lyotropic liquid crystal, to determine the osmotic pressure and the solvent chemical potential as functions of polymer concentration. The resulting data were used to test the validity of the theory of Khokhlov and Semenov of polymer liquid crystals, which was almost quantitative in predicting the isotropicliquid crystal phase diagrams for reasonably stiff polymers. It was found that the second virial approximation with respect to free energy basic to this theory was only valid under limited conditions and the scaled particle theory of Cotter on spherocylinders was shown to be a promising alternative.
\end{abstract}

KEY WORDS Stiff-Chain Polymer / Chemical Potential / Osmotic Pressure / Sedimentation Equilibrium / Liquid Crystal / Phase Diagram / Onsager Theory / Concentrated Solution /

In a previous publication ${ }^{1}$ we showed that the theory of Khokhlov and Semenov (KS theory) for wormlike cylinders ${ }^{2,3}$ gives an almost quantitative explanation of experimental phase diagrams for binary systems of poly(hexyl isocyanate) (PHIC)-toluene and of schizophyllan (a triple-helical polysaccharide)water, when the geometric parameters of the respective polymers determined from dilute solution properties are used. However, an appreciable discrepancy between the KS theory and experiment was found in the phase diagram of the dichloromethane (DCM) solutions of PHIC characterized by the smallest persistence length and the highest phase boundary concentrations among the systems investigat- ed. This discrepancy may be due to the second virial approximation to free energy which is used in the KS theory. To test the validity of the second virial approximation more directly, other thermodynamic properties, for example, the osmotic pressure should be measured for the systems studied before. For this reason, we determined the chemical potential of the solvent for solutions of PHIC in DCM at $20^{\circ} \mathrm{C}$, as well as the osmotic pressure for solutions of PHIC in toluene at $25^{\circ} \mathrm{C}$, both in the isotropic state. In this publication, we present the results from these experiments and use them to test the basis of the KS theory, namely, the second virial approximation.

\footnotetext{
† To whom all correspondence should be addressed.
} 


\section{EXPERIMENTAL}

Six fractionated samples of poly(hexyl isocyanate) (PHIC) were taken from our stock. ${ }^{4}$ They are nearly monodisperse in molecular weight, with the polydispersity index $\bar{M}_{z} / \bar{M}_{w}$ smaller than 1.11 , as shown in Table I.

Osmotic pressures $\Pi$ of PHIC fractions $\mathbf{J} 2$ 2, $\mathrm{K}-2$, and NRX-12 in toluene at $25^{\circ} \mathrm{C}$ were measured using a Knauer osmometer with a Sartorius SM11536 membrane at polymer weight fractions $w$ below 0.1 .

Sedimentation equilibrium measurement was made on solutions of PHIC fractions Z-2, L-2, NRX-12, and Z-20 in dichloromethane $(\mathrm{DCM})$ at $20^{\circ} \mathrm{C}$. A Beckman-Spinco model E analytical ultracentrifuge equipped with a schrielen optical system was used following the procedure previously established. ${ }^{5}$

For a binary solution consisting of a monodisperse polymer and a solvent, the equilibrium condition for the solvent chemical potential at the radial distance $r$ from the center of rotation is expressed as

$$
\partial \Delta \mu_{0} / \partial w=\frac{M_{0} w(r) r \omega^{2}(\partial v / \partial w)(\partial n / \partial w)}{v(d n / d r)}
$$

Here, $M_{0}$ and $\Delta \mu_{0}$ are the molecular weight and excess chemical potential (per mol) of the solvent component, respectively, $v$ the specific volume of the solution, $\omega$ the angular velocity, $\partial n / \partial w$ the refractive index increment on the $w$ basis, and $w(r)$ the polymer weight fraction at $r$. Values of the refractive index gradient $d n / d r$ were determined as a function of $r$ from sedimentation equilibrium measurements and used to compute $\partial \Delta \mu_{0} / \partial w$ by eq 1 .

The specific volumes $v$ needed for the analysis were measured on concentrated solutions of PHIC in DCM at $20^{\circ} \mathrm{C}$, using a LipkinDevison type pycnometer of $5 \mathrm{~cm}^{3}$ capacity. Figure 1 shows the plot of $v$ against polymer weight fraction $w$. The data points are fitted by the indicated line, which is represented by

$$
v=0.7542+0.2378 w+0.0343 w^{2}
$$

The intercept and initial slope of the line are consistent with the solvent density and the partial specific volume $v_{\mathrm{p}}$ of PHIC in DCM at $20^{\circ} \mathrm{C}$ determined from measurements on dilute

Table I. Molecular weights of the PHIC samples investigated ${ }^{4}$

\begin{tabular}{lccc}
\hline Sample & $\bar{M}_{w} / 10^{3}$ & $\bar{M}_{n} / 10^{3}$ & $\bar{M}_{z} / \bar{M}_{w}$ \\
\hline Z-2 & 20.9 & - & 1.06 \\
J2-2 & $32.0^{\mathrm{a}}$ & 30.8 & 1.11 \\
L-2 & 40.7 & - & 1.06 \\
K-2 & 68 & 66.4 & 1.02 \\
NRX-12 & 133 & 122 & 1.05 \\
Z-20 & 244 & - & 1.05 \\
\hline
\end{tabular}

${ }^{\text {a }}$ Viscosity-average molecular weight obtained from $[\eta]$ in toluene at $25^{\circ} \mathrm{C}$.

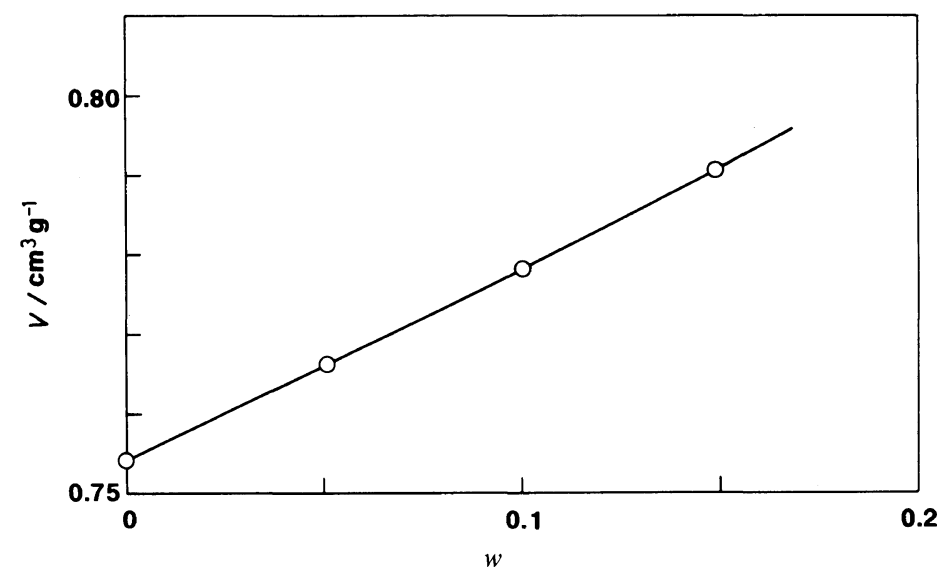

Figure 1. Specific volume $v$ of DCM solutions of PHIC at $20^{\circ} \mathrm{C}$ as a function of $w$. 
solutions. ${ }^{1,4}$

A modified Schultz-Cantow differential refractometer was used to measure the excess refractive index $\Delta n$ of a solution over another solution (or the solvent) for PHIC in DCM at $20^{\circ} \mathrm{C}$ with light of $546 \mathrm{~nm}$ wavelength. Figure 2 shows $\Delta n / \Delta w$ plotted against $w_{\mathrm{Av}}$, where $\Delta w$ is the small difference in $w$ between the two solu-

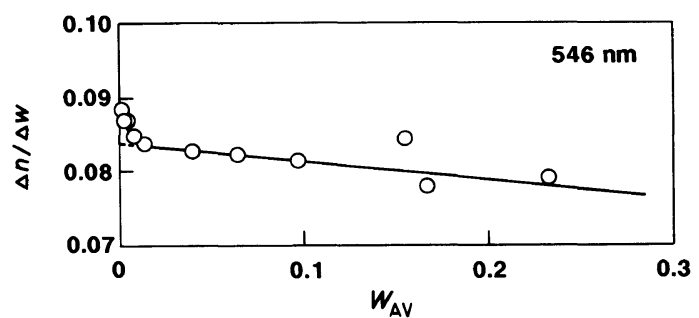

Figure 2. Plots of $\Delta n / \Delta w v s . w_{\mathrm{Av}}$ for DCM solutions of PHIC at $20^{\circ} \mathrm{C}$ and $546 \mathrm{~nm}$. tions (or the solution and the solvent) and $w_{\mathrm{AV}}$ the average of $w$ for them. For $w_{\mathrm{Av}}>0.01$, it can be seen that $\Delta n / \Delta w$ linearly decreases with $w_{\mathrm{Av}}$. Regarding $w_{\mathrm{Av}}$ and $\Delta n / \Delta w$ as $w$ and $\partial n / \partial w$, respectively, we obtained the equation

$$
\partial n / \partial w=-0.02432 w+0.08386
$$

Solutions were prepared by mixing appropriate amounts of a given fraction and a solvent and their $w$ were determined gravimetrically. Their mass concentrations $c$ were obtained from $c=w / v$, with $v$ being given by eq 2 for DCM solutions and $v=0.987 w+(1-$ $w) / 0.86231$ for toluene solutions; the partial specific volume $v_{\mathrm{p}}$ of PHIC was taken to be $0.987 \mathrm{~cm}^{3} \mathrm{~g}^{-1}$ in toluene at $25^{\circ} \mathrm{C}$ and 0.992 $\mathrm{cm}^{3} \mathrm{~g}^{-1}$ in $\mathrm{DCM}$ at $20^{\circ} \mathrm{C}$. ${ }^{1,4}$ The partial de-

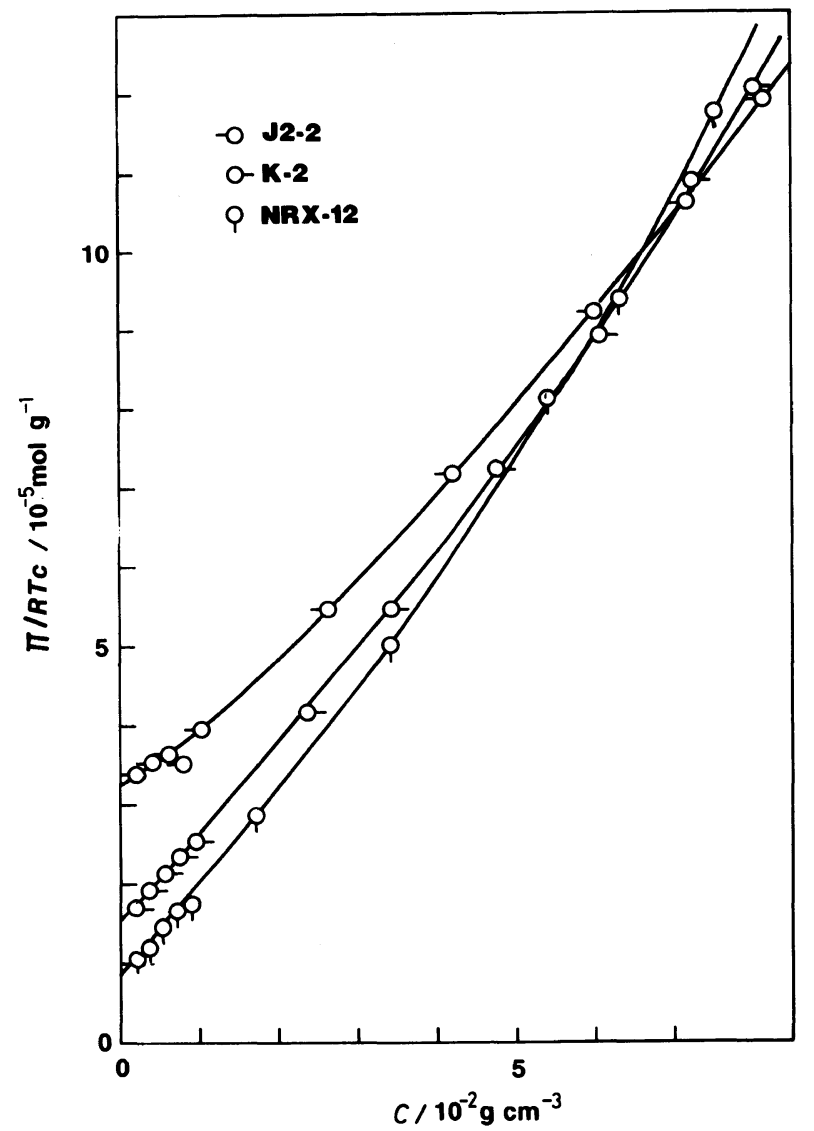

Figure 3. Plots of $\Pi / c R T$ vs. $c$ for PHIC in toluene at $25^{\circ} \mathrm{C}$. (-O), J2-2; (○-), K-2; (Q), NRX-12. 


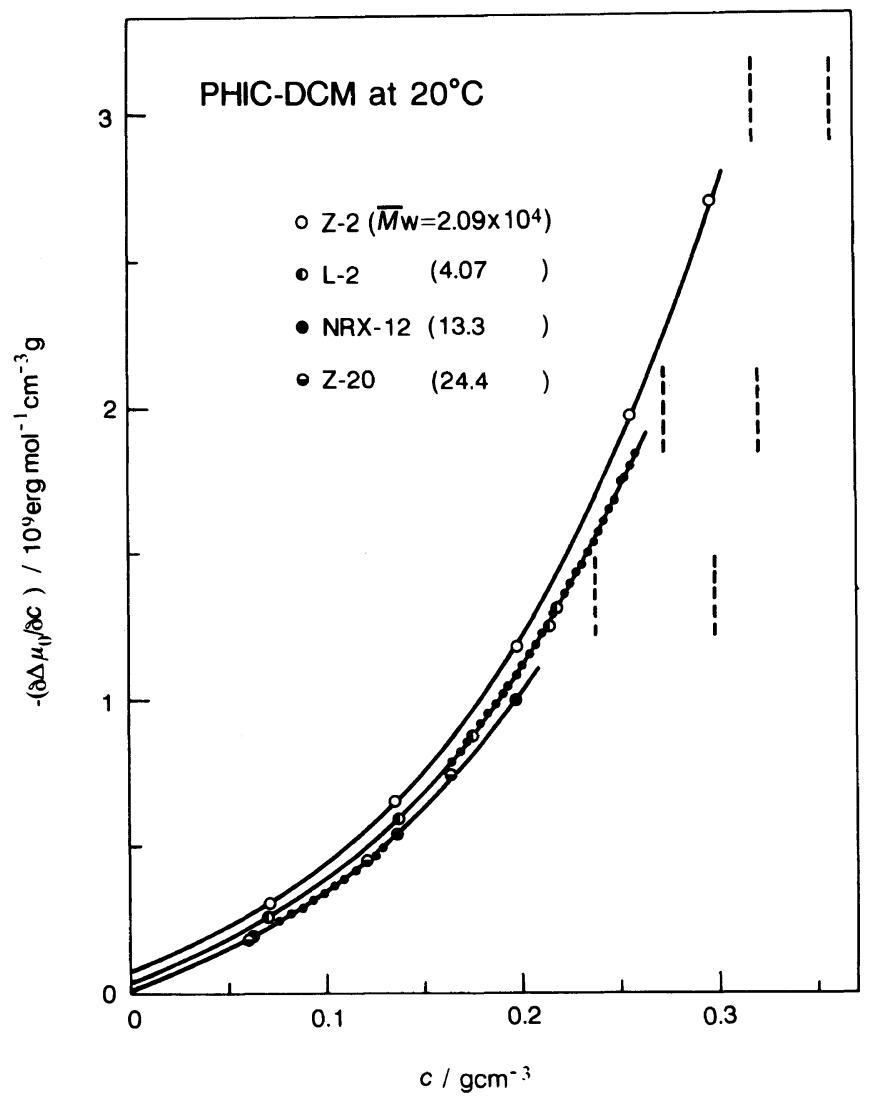

Figure 4. Plots of $-\left(\partial \Delta \mu_{0} / \partial c\right) v s . c$ for DCM solutions of PHIC at $20^{\circ} \mathrm{C} ;(\bigcirc), \mathrm{Z}-2 ;(\bigcirc), \mathrm{L}-2 ;(\bigcirc), \mathrm{NRX}-$ $12 ;(\Theta), \mathrm{Z}-20$; dots, the data obtained at high rotor speeds with long-solution columns; vertical dashed lines, phase boundaries determined for samples Z-2, L-2, and NRX-12.

rivative of $\Delta \mu_{0}$ with respect to $c, \partial \Delta \mu_{0} / \partial c$, was computed from $\partial \Delta \mu_{0} / \partial w$ using the relation

$$
\partial \Delta \mu_{0} / \partial c=\left(\partial \Delta \mu_{0} / \partial w\right)(\partial w / \partial c)
$$

\section{RESULTS}

\section{Osmotic Pressure}

All the osmotic pressure data are summarized in Table II, where the data used for determining $\bar{M}_{n}$ taken from the previous paper $^{4}$ are also included. Figure 3 shows plots of $\Pi / c R T$ versus mass concentration $c$ for the three samples, J2-2, K-2, and NRX-12, where $R T$ has the usual meaning. The intercept and initial slope of each indicated line correspond to $\bar{M}_{n}{ }^{-1}$ and $A_{2}$, respectively. It can be seen that the indicated lines rise monotonically with $c$ and cross one another at $c$ about 0.065 $\mathrm{g} \mathrm{cm}^{-3}$.

\section{Sedimentation Equilibrium}

When the rotor speed was low and the solution column was short, the concentration gradient developed in the cell was almost constant throughout the entire solution, and $w(r)$ at the midpoint of the solution was very close to the weight fraction in the original solution. The data for the short solution columns are summarized in Table III. They are shown as plots of $\partial \Delta \mu_{0} / \partial c v s . c$ in Figure 4. The data points for each sample are fitted by 
Table II. Osmotic pressures of toluene solutions of PHIC at $25^{\circ} \mathrm{C}$

\begin{tabular}{|c|c|c|c|}
\hline \multirow{2}{*}{$10^{2} w$} & $c$ & $\Pi$ & $\Pi(c R T)^{-1}$ \\
\hline & $10^{-2} \mathrm{~g} \mathrm{~cm}^{-3}$ & $10^{5}{\mathrm{dyn} \mathrm{cm}^{-2}}^{-2}$ & $10^{-5} \mathrm{~mol} \mathrm{~g}^{-1}$ \\
\hline \multicolumn{4}{|c|}{$\mathrm{J} 2-2$} \\
\hline 0.2854 & 0.2099 & 0.01758 & 3.378 \\
\hline 0.5774 & 0.4246 & 0.03705 & 3.520 \\
\hline 0.8466 & 0.6225 & 0.05587 & 3.620 \\
\hline 1.1079 & 0.8147 & 0.07077 & 3.504 \\
\hline 1.4282 & 1.0502 & 0.10280 & 3.948 \\
\hline 3.055 & 2.646 & 0.3579 & 5.455 \\
\hline 4.868 & 4.228 & 0.7522 & 7.176 \\
\hline 6.890 & 6.003 & 1.3720 & 9.219 \\
\hline 8.212 & 7.169 & 1.8852 & 10.607 \\
\hline 9.325 & 8.154 & 2.409 & 11.917 \\
\hline \multicolumn{4}{|c|}{$\mathrm{K}-2$} \\
\hline 0.2603 & 0.1914 & 0.00791 & 1.667 \\
\hline 0.5244 & 0.3856 & 0.01819 & 1.903 \\
\hline 0.7818 & 0.5749 & 0.03026 & 2.123 \\
\hline 1.045 & 0.7681 & 0.04456 & 2.340 \\
\hline 1.320 & 0.9710 & 0.06096 & 2.533 \\
\hline 2.742 & 2.374 & 0.2448 & 4.159 \\
\hline 3.973 & 3.446 & 0.4666 & 5.461 \\
\hline 5.502 & 4.783 & 0.8575 & 7.231 \\
\hline 6.969 & 6.073 & 1.345 & 8.933 \\
\hline 8.313 & 7.258 & 1.961 & 10.897 \\
\hline 9.195 & 8.039 & 2.404 & 12.060 \\
\hline \multicolumn{4}{|c|}{ NRX-12 } \\
\hline 0.2948 & 0.2168 & 0.00566 & 1.052 \\
\hline 0.5071 & 0.3729 & 0.01110 & 1.200 \\
\hline 0.7454 & 0.5481 & 0.01971 & 1.450 \\
\hline 1.0019 & 0.7367 & 0.03047 & 1.668 \\
\hline 1.2723 & 0.9356 & 0.04034 & 1.739 \\
\hline 1.9933 & 1.724 & 1.222 & 2.859 \\
\hline 3.950 & 3.426 & 0.4261 & 5.017 \\
\hline 6.227 & 5.419 & 1.094 & 8.141 \\
\hline 7.262 & 6.331 & 1.475 & 9.395 \\
\hline 8.634 & 7.542 & 2.199 & 11.758 \\
\hline
\end{tabular}

a smooth curve starting from the value of $R T V_{0}^{\circ} / \bar{M}_{w}$ at $c=0$, where $V_{0}^{\circ}$ is the molar volume of the solvent. The curve for sample Z-20 almost overlaps with that for sample NRX-12.

When a higher rotor speed was used with a long solution columns, a larger concentration gradient was established at sedimentation equilibrium. Actually, long solution column experiments yielded non-linear concentration gradients. In the analysis, each solution was divided into a number of short columns at different $r$, and $w(r)$ was determined so that the mass conservation held over the entire solution. For each $r, \partial \Delta \mu_{0} / \partial c$ value was calculated from the observed values of $w(r)$ and $d n / d r$. The $w(r)$ and $\partial \Delta \mu_{0} / \partial c$ values thus determined are summarized in Table IV. These data are shown in Figure 4 by dots. It can be seen that the two types of data points for each sample follow closely a smooth composite curve irrespective of rotor speed, indicating that there is no molecular weight fractionation on centrifugation as expected from the samples' monodispersity.

Each of the indicated curves rises steeply with $c$ up to the phase boundary concentrations (vertical dashed lines). The initial slope of the curve corresponds to the $A_{2}$ term and the steep increase of $-\partial \Delta \mu_{0} / \partial c$ with $c$ is due to the contribution of the higher order terms in $c$.

\section{DISCUSSION}

\section{Thermodynamic Properties of Isotropic Solu- tions}

The Khokhlov-Semenov theory of persistent chains ${ }^{2,3}$ uses the same expression of osmotic pressure for isotropic solutions as that of the Onsager theory, ${ }^{6}$ which reads

$$
\Pi / k T=c^{\prime}+(\pi / 4) L^{2} d c^{\prime 2}
$$

where $c^{\prime}, L$, and $d$ are the number concentration, the contour length, and the diameter of the polymer, respectively. Thus, the excess chemical potential $\Delta \mu_{0}$ of the solvent as a function of $c$ is expressed as

$$
-\Delta \mu_{0} /\left(V_{0}^{\circ} R T\right)=\Pi / R T=c\left(1 / M+A_{2} c\right)
$$

where $A_{2}$ is given by

$$
A_{2}=(\pi / 4) N_{\mathrm{A}} d M_{\mathrm{L}}{ }^{-2}
$$

and $M_{\mathrm{L}}$ is the molar mass per unit contour length of the polymer. The effect of finite rod 
T. ITOU et al.

Table III. Sedimentation equilibrium data for PHIC in DCM at $20^{\circ} \mathrm{C}$

\begin{tabular}{|c|c|c|c|c|}
\hline \multirow{2}{*}{$w$} & $c$ & \multirow{2}{*}{$\frac{\text { Rotor speed }}{\mathrm{rpm}}$} & \multirow{2}{*}{$\frac{-\mathrm{d} n / \mathrm{d} c}{10^{-3} \mathrm{~cm}^{-1}}$} & \multirow{2}{*}{$\frac{-\left[\partial \Delta \mu_{0} / \partial c\right]}{\mathrm{J} \mathrm{mol}^{-1} \mathrm{~cm}^{3} \mathrm{~g}^{-1}}$} \\
\hline & $\mathrm{g} \mathrm{cm}^{-3}$ & & & \\
\hline \multicolumn{5}{|c|}{$\mathrm{Z}-2$} \\
\hline 0.0542 & 0.0706 & 15843 & 5.93 & 30.7 \\
\hline 0.1055 & 0.1353 & 15847 & 5.49 & 65.4 \\
\hline 0.1569 & 0.1980 & 15852 & 4.57 & 118.0 \\
\hline 0.2063 & 0.2564 & 15875 & 3.72 & 196.4 \\
\hline 0.2422 & 0.2976 & 15731 & 3.16 & 269.3 \\
\hline \multicolumn{5}{|c|}{ L-2 } \\
\hline 0.0540 & 0.0704 & 10001 & 2.79 & 25.9 \\
\hline 0.1072 & 0.1374 & 10001 & 2.45 & 59.7 \\
\hline 0.1374 & 0.1745 & 10003 & 2.16 & 87.1 \\
\hline 0.1704 & 0.2141 & 9999 & 1.87 & 125.1 \\
\hline 0.1737 & 0.2181 & 9993 & 1.83 & 130.8 \\
\hline \multicolumn{5}{|c|}{ NRX-12 } \\
\hline 0.0483 & 0.0631 & 6351 & 1.33 & 19.6 \\
\hline 0.1061 & 0.1361 & 6349 & 1.08 & 54.0 \\
\hline 0.1557 & 0.1966 & 6328 & 0.868 & 99.9 \\
\hline \multicolumn{5}{|c|}{$\mathrm{Z}-20$} \\
\hline 0.0459 & 0.0600 & 11959 & 4.83 & 18.0 \\
\hline 0.0936 & 0.1205 & 13910 & 5.44 & $44.8^{\circ}$ \\
\hline 0.1287 & 0.1639 & 10020 & 2.36 & 75.4 \\
\hline
\end{tabular}

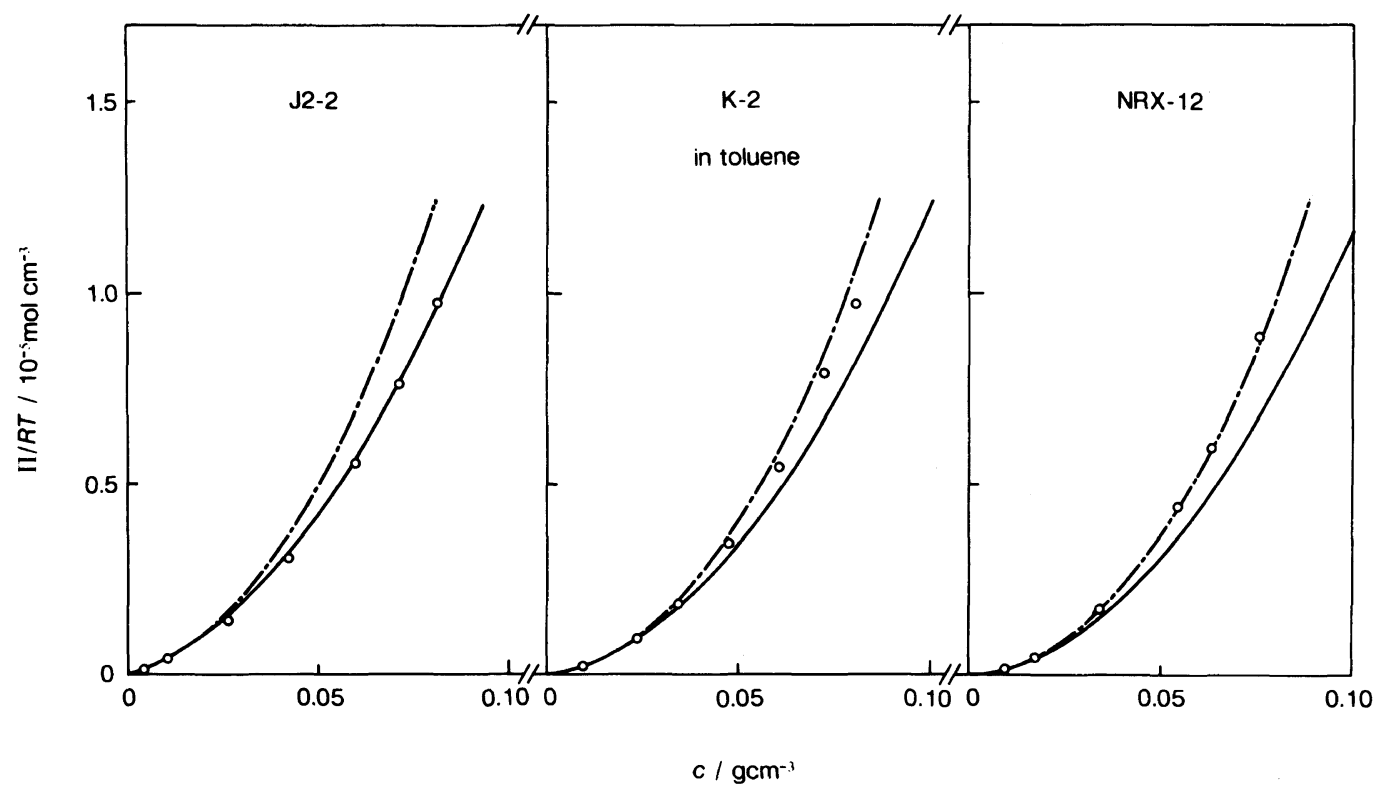

Figure 5. Plots of $\Pi / R T$ vs. $c$ for PHIC in toluene at $25^{\circ} \mathrm{C}$. Solid lines, the Onsager theory; ${ }^{6}$ dash-dot lines, the Cotter theory. ${ }^{9}$ 
Concentrated Solutions of Poly(hexyl isocyanate)

Table IV. Sedimentation equilibrium data for PHIC in DCM at $20^{\circ} \mathrm{C}$

\begin{tabular}{|c|c|c|c|c|}
\hline$r$ & $-\mathrm{d} n / \mathrm{d} r$ & & $c(r)$ & $-\left(\partial \Delta \mu_{0} / \partial c\right)$ \\
\hline $\mathrm{cm}$ & $10^{-3} \mathrm{~cm}^{-1}$ & & $\mathrm{~g} \mathrm{~cm}^{-3}$ & $\mathrm{~J} \mathrm{~mol}^{-1} \mathrm{~cm}^{3} \mathrm{~g}^{-1}$ \\
\hline \multicolumn{5}{|c|}{ Sample L- $2 ; w=0.1704 ;$ rotor speed $=29788 \mathrm{rpm}$} \\
\hline 6.813 & 13.55 & 0.2079 & 0.2582 & 183.4 \\
\hline 6.826 & 13.73 & 0.2057 & 0.2556 & 179.4 \\
\hline 6.839 & 13.91 & 0.2034 & 0.2530 & 175.4 \\
\hline 6.852 & 13.88 & 0.2012 & 0.2504 & 174.1 \\
\hline 6.864 & 14.25 & 0.1989 & 0.2478 & 167.8 \\
\hline 6.877 & 14.41 & 0.1966 & 0.2451 & 164.3 \\
\hline 6.890 & 14.56 & 0.1943 & 0.2423 & 160.7 \\
\hline 6.902 & 14.74 & 0.1920 & 0.2396 & 157.0 \\
\hline 6.915 & 14.93 & 0.1896 & 0.2368 & 153.3 \\
\hline 6.928 & 15.11 & 0.1872 & 0.2340 & 149.8 \\
\hline 6.941 & 15.30 & 0.1847 & 0.2311 & 146.1 \\
\hline 6.953 & 15.44 & 0.1823 & 0.2282 & 142.9 \\
\hline 6.966 & 15.65 & 0.1798 & 0.2253 & 139.3 \\
\hline 6.979 & 15.85 & 0.1773 & 0.2223 & 135.8 \\
\hline 6.991 & 16.04 & 0.1747 & 0.2193 & 132.4 \\
\hline 7.004 & 16.24 & 0.1721 & 0.2162 & 129.0 \\
\hline 7.017 & 16.47 & 0.1695 & 0.2131 & 125.3 \\
\hline 7.030 & 16.65 & 0.1669 & 0.2100 & 122.2 \\
\hline 7.042 & 16.90 & 0.1642 & 0.2068 & 118.5 \\
\hline 7.055 & 17.15 & 0.1615 & 0.2035 & 115.0 \\
\hline 7.068 & 17.45 & 0.1587 & 0.2002 & 111.3 \\
\hline 7.080 & 17.65 & 0.1560 & 0.1969 & 108.2 \\
\hline 7.093 & 17.90 & 0.1531 & 0.1934 & 104.9 \\
\hline 7.106 & 18.16 & 0.1503 & 0.1900 & 101.5 \\
\hline 7.119 & 18.41 & 0.1474 & 0.1866 & 98.3 \\
\hline 7.131 & 18.65 & 0.1444 & 0.1830 & 95.1 \\
\hline 7.144 & 18.94 & 0.1415 & 0.1794 & 91.9 \\
\hline 7.157 & 19.22 & 0.1385 & 0.1757 & 88.7 \\
\hline 7.169 & 19.50 & 0.1354 & 0.1721 & 85.5 \\
\hline 7.182 & 19.85 & 0.1323 & 0.1682 & 82.1 \\
\hline 7.195 & 20.26 & 0.1291 & 0.1644 & 78.6 \\
\hline \multicolumn{5}{|c|}{ Sample NRX-12; $w=0.08001 ;$ rotor speed $=15872 \mathrm{rpm}$} \\
\hline 6.725 & 6.634 & 0.1008 & 0.1294 & 49.2 \\
\hline 6.749 & 6.708 & 0.0988 & 0.1270 & 48.0 \\
\hline 6.772 & 6.776 & 0.0969 & 0.1246 & 46.7 \\
\hline 6.796 & 6.859 & 0.0949 & 0.1222 & 45.3 \\
\hline 6.819 & 6.921 & 0.0929 & 0.1197 & 44.0 \\
\hline 6.843 & 6.991 & 0.0909 & 0.1171 & 42.9 \\
\hline 6.866 & 7.040 & 0.0889 & 0.1146 & 41.7 \\
\hline 6.889 & 7.119 & 0.0869 & 0.1121 & 40.4 \\
\hline 6.913 & 7.194 & 0.0848 & 0.1095 & 39.2 \\
\hline 6.936 & 7.271 & 0.0828 & 0.1069 & 37.9 \\
\hline 6.960 & 7.362 & 0.0807 & 0.1042 & 36.6 \\
\hline 6.983 & 7.462 & 0.0785 & 0.1016 & 35.2 \\
\hline 7.007 & 7.515 & 0.0764 & 0.0989 & 34.1 \\
\hline 7.030 & 7.635 & 0.0742 & 0.0962 & 32.7 \\
\hline 7.054 & 7.688 & 0.0720 & 0.0933 & 31.6 \\
\hline 7.077 & 7.794 & 0.0698 & 0.0905 & 30.3 \\
\hline 7.101 & 7.884 & 0.0676 & 0.0877 & 29.1 \\
\hline 7.124 & 7.930 & 0.0653 & 0.0849 & 28.0 \\
\hline 7.148 & 7.941 & 0.0630 & 0.0820 & 27.1 \\
\hline 7.171 & 8.028 & 0.0608 & 0.0790 & 25.9 \\
\hline 7.195 & 8.108 & 0.0585 & 0.0761 & 24.7 \\
\hline
\end{tabular}




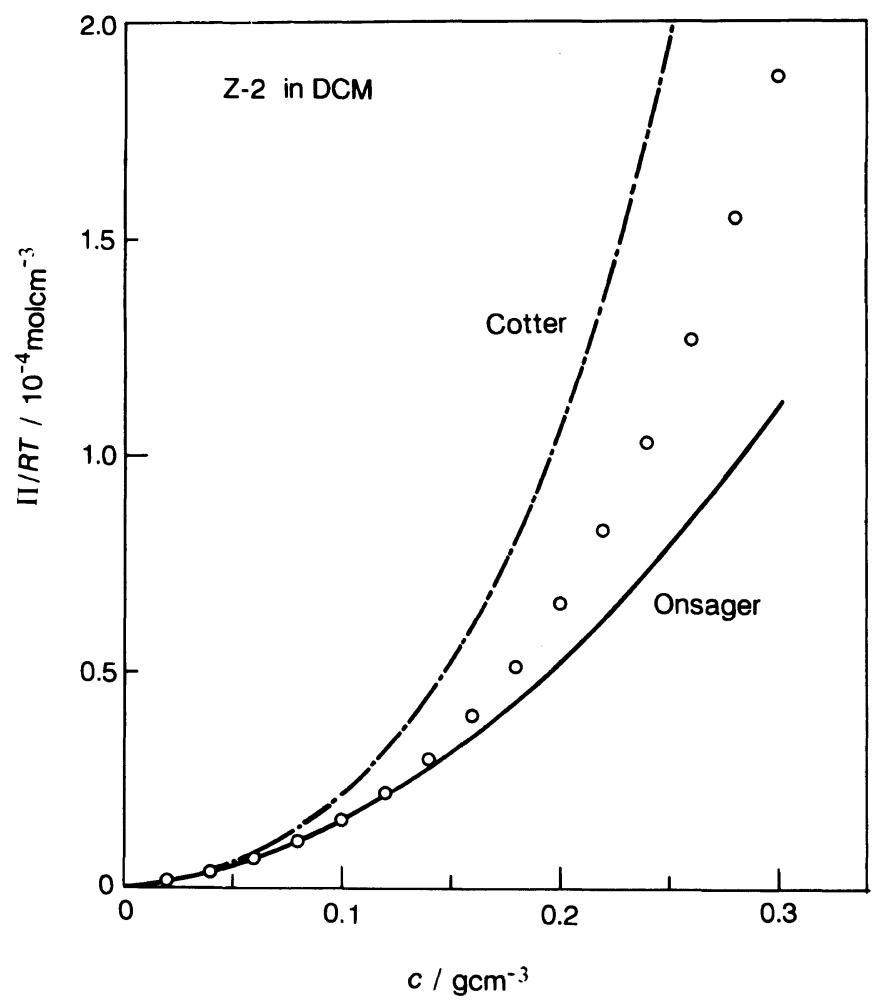

Figure 6. $\Pi / R T$ vs. $c$ for sample Z-2 in DCM determined by sedimentation equilibrium; circles, calculated by integrating $\partial \Delta \mu_{0} / \partial c$ for DCM solutions of PHIC sample Z-2 with respect to $c$; solid curve, the Onsager theory; ${ }^{6}$ dash-dot curve, the Cotter theory. ${ }^{9}$

length on $A_{2}^{7,8}$ is neglected in these equations. Cotter $^{9}$ modified the Onsager theory on the basis of the scaled particle theory to obtain an expression for $\Pi$ which is expressed in terms of $L, d$, and $c$. Her theory agrees with the Onsager theory at the limit of low $c$, and reduces to the scaled particle theory for spheres ${ }^{10}$ at $L / d=1$. We use both the Onsager and Cotter equations in analyzing the osmotic pressure and sedimentation data on PHIC with the expectation that the excluded volume would be the same for semiflexible polymers as that for rods. ${ }^{2,3,8}$

Figure 5 shows plots of $\Pi / R T$ against $c$ for PHIC in toluene at $25^{\circ} \mathrm{C}$, where the solid and dash-dot curves represent the values predicted by the Onsager theory and by the Cotter theory, respectively; $d$ is taken to be $1.25 \mathrm{~nm}$ to be consistent with the measured values of $v_{\mathrm{p}}$ and $L=M / M_{\mathrm{L}}$, with $M=\bar{M}_{n}$ or $\bar{M}_{w}$ for osmot- ic pressure or sedimentation data, respectively; $M_{\mathrm{L}}=740 \mathrm{~nm}^{-1}$. It can be seen that the data points for samples K-2 and NRX-12 appear definitely above the Onsager curve and rather close to the Cotter curve. On the other hand, those for sample J2-2 appear to follow accurately the Onsager curve within the range of $c$ investigated. However, it is premature to look for a definite reason for the difference between J2-2 and the other samples owing to the broader molecular weight distribution for J2-2.

The value of $\Delta \mu_{0}$ can be evaluated also from integration of $\partial \Delta \mu_{0} / \partial c$ by $c$ and conversion to $\Pi$ by $\Pi=-\Delta \mu_{0} / V_{0}^{\circ}$. The data for DCM solutions were treated in this way. Figure 6 compares the values of $\Pi / R T$ estimated for the system $\mathrm{Z}-2+\mathrm{DCM}$ with the predictions of the two theories mentioned above; the ex- 


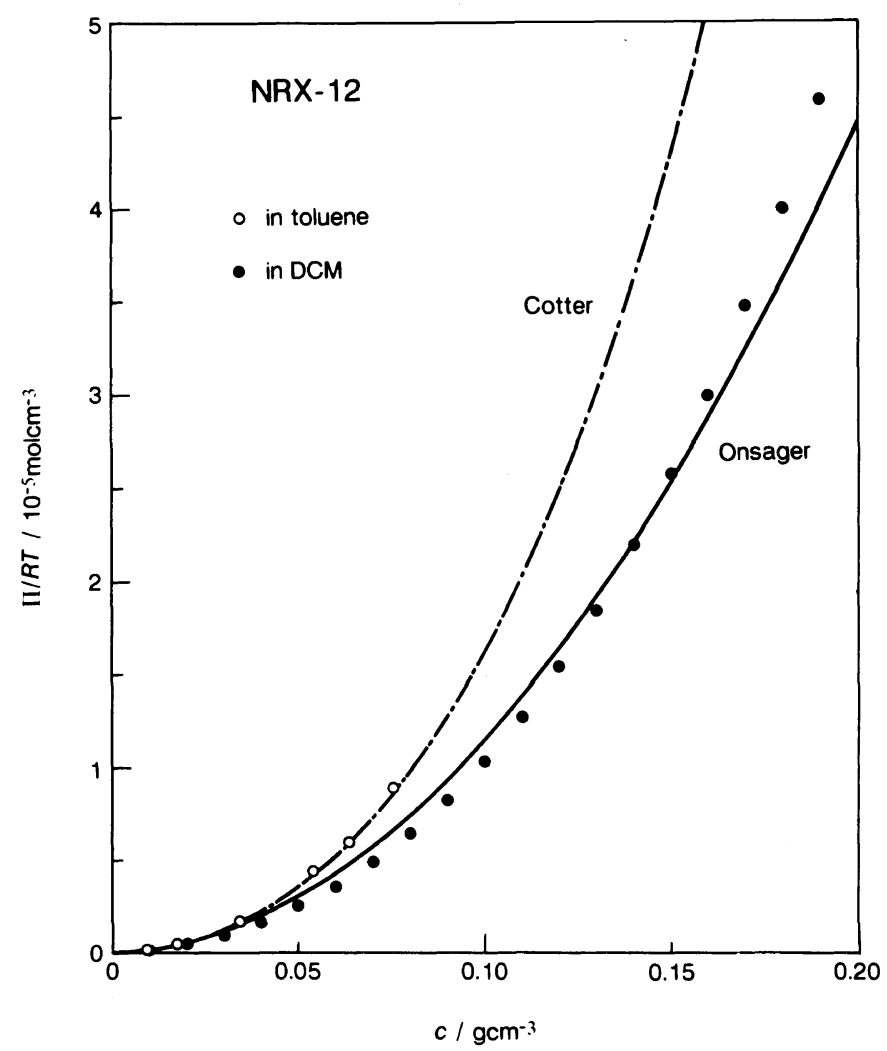

Figure 7. $\Pi / R T$ vs. $c$ for sample NRX-12: open circles, osmotic pressure data in toluene at $25^{\circ} \mathrm{C}$; filled circles, sedimentation equilibrium data in DCM at $20^{\circ} \mathrm{C}$. Theoretical values: solid curve, the Onsager theory, ${ }^{6}$ dash-dot curve, the Cotter theory. ${ }^{9}$

perimental data are shown by circles, which represent the integral values at the indicated $c$. The data points follow the Onsager curve up to $c$ of $0.13 \mathrm{~g} \mathrm{~cm}^{-3}$, but tend to deviate appreciably above it with increasing $c$. The Cotter curve is seen considerably above the experimental one at any value of $c$. At the isotropic-biphasic phase boundary $c$ of 0.317 $\mathrm{g} \mathrm{cm}^{-3}$, the experimental value of $\Pi / R T$ is larger than the Onsager one by a factor of 1.6. A similar trend was found for the other samples in DCM.

Figure 7 compares the data for $\Pi / R T$ of toluene at $25^{\circ} \mathrm{C}$ obtained by osmometry with those for DCM at $20^{\circ} \mathrm{C}$ obtained by sedimentation equilibrium, both with sample NRX-12; the prediction by the Onsager theory (solid curve) and that by the Cotter theory (dash-dot curve) are also shown. It is seen that the increase in $\Pi / R T$ with $c$ is steeper for toluene than for DCM. This difference suggests that the polymer-solvent interaction plays an important role in the solvent chemical potential and phase diagram for a semiflexible polymer.

In the above analysis of the sedimentation data, we took the diameter $d$ to be $1.25 \mathrm{~nm}$ obtained from the partial specific volume $v_{\mathrm{p}}$ of PHIC approximately equal to its hard-core volume, and calculated $A_{2}$ and related quantities. However, $A_{2}$ is actually related to the polymer-polymer interaction in the solution and needs not be consistent with the hard-core volume. Thus in either theory $d$ may be treated as an adjustable parameter expressing this interaction. Indeed, we found that the Cotter theory can describe the data for the DCM 
solutions if $d$ is taken to be $1.0 \mathrm{~nm}$. It must be noted that the Flory theory of rodlike polymers ${ }^{11}$ does not give quantitative description of the present osmotic pressure data as well as those for phase diagrams. ${ }^{1,4,5,12,13}$

We may summarize these discussions as follows. For both the DCM and toluene solutions of PHIC, the Onsager theory predicts rather quantitatively the experimental osmotic pressure and solvent chemical potential data only at low $c$, but tends to underestimate them at moderately high $c$. This clearly shows that, with regard to the solvent chemical potential and osmotic pressure, the second virial approximation employed in the Onsager approach is not valid a near the isotropic-liquid crystal phase boundary concentrations. Thus it follows that the theory of Khokhlov and Semenov, ${ }^{2,3}$ which uses the same expression for $\Pi$ as eq 5 , is still approximate in spite of the good agreement between theory and experiment in the phase boundary concentrations found for PHIC in toluene and aqueous schizophyllan. ${ }^{1}$ The agreement in the phase boundaries of the two systems may be attributed to a cancellation of the third and higher virial terms in the phase coexistence equations. We conclude from these discussions that consideration of the terms in $c$ higher than $c^{3}$ is essential toward a more quantitative discussion of the thermodynamic properties of semiflexible polymer solutions and suggest that use of the Cotter theory is a promising alternative.
However a direct application of the Cotter theory does not give a consistent explanation of the chemical potential and phase diagram data $^{5,12,13}$ because of the finite stiffness of the polymers investigated. Attempts have been made to estimate the third virial coefficient of rods ${ }^{7}$ and to calculate the phase diagrams for solutions of rods. ${ }^{8}$ However, so far no such theoretical attempt has been reported for semiflexible polymers. Therefore we defer further quantitative analysis of the data for future work. $^{14}$

\section{REFERENCES}

1. T. Itou and A. Teramoto, Macromolecules, 21, 2225 (1988).

2. A. R. Khokhlov and A. N. Semenov, Physica $A$ (Amsterdam), 108A, 546 (1981).

3. A. R. Khokhlov and A. N. Semenov, Physica $A$ (Amsterdam), 112A, 605 (1982).

4. T. Itou, H. Chikiri, and A. Teramoto, Polym. J., 20, 143 (1988).

5. K. Van and A. Teramoto, Polym. J., 17, 409 (1985).

6. L. Onsager, Ann. N. Y. Acad. Sci., 51, 627 (1947).

7. A. P. Straley, Mol. Cryst. Liq. Cryst., 24, 37 (1973).

8. T. Odijk, Macromolecules, 19, 2313 (1986).

9. A. J. Cotter, J. Chem. Phys., 66, 1098 (1977).

10. H. Reiss, H. L. Frisch, and J. L. Lebowitz, J. Chem. Phys., 31, 369 (1959).

11. P. J. ,Flory, Proc. R. Soc. London, Ser. A, 234, 73 (1956).

12. K. Kubo and K. Ogino, Polymer, 16, 629 (1975).

13. K. Kubo and K. Ogino, Mol. Cryst. Liq. Cryst., 53, 207 (1979).

14. T. Sato and A. Teramoto, in preparation. 